\title{
Modifications to the von Laue Statistical Distribution of the Times to Breakdown at a Polymer-Oil Interface
}

\author{
Martin J. Given, Mark P. Wilson, Igor V. Timoshkin, Scott J. MacGregor, Tao Wang \\ Department of Electronic and Electrical Engineering, \\ University of Strathclyde, \\ Glasgow G1 1XW, UK \\ Mark A. Sinclair, Ken J. Thomas \\ AWE Aldermaston, \\ Reading, RG7 4PR, UK \\ and Jane M. Lehr \\ Department of Electrical and Computer Engineering, \\ University of New Mexico \\ Albuquerque, NM 87131, USA
}

\begin{abstract}
A statistical analysis has been undertaken to determine the statistical and formative times associated with breakdowns along a polymer-oil interface under impulse conditions. Early analysis was based on an assumption that the breakdown data followed the von Laue Distribution. However, it was found that in the Laue plots there were deviations from the expected straight line behavior at short times to breakdown, which may be due to a normal distribution in values of the formative times. In addition, the plots showed multiple straight line sections, which suggested that changes were occurring to the breakdown processes during the experimental run, or that more than one mechanism of breakdown was occurring. Values of the statistical time $t_{s}$ and the formative time $t_{f}$ were determined from the data by making choices on the straight line section to be considered, and ignoring the effects of the normal distribution on the derived values of $t_{s}$ and $t_{f}$. The present paper is focused on further development of this statistical method, including a rigorous analysis of the experimental data, taking into account the effect that a normal distribution of the formative times has on the derived values of $t_{s}$ and $t_{f}$. Optimal fits in terms of three parameters: $t_{s}, t_{f}$, and $\sigma_{f}$ (the standard deviation of the formative time) have been derived using Kolmogorov-Smirnov statistics to quantify the quality of fit. The quality of these fits and the applicability of this approach is discussed.
\end{abstract}

Index Terms - Dielectric breakdown, flashover, pulsed power supplies, statistical analysis.

\section{INTRODUCTION}

TO design and manufacture pulsed power machines with higher energy densities, either by increasing the output voltage or making the machine's footprint more compact, information on the time to breakdown of system components is required. This behavior will be affected by the peak value of the impulse voltage experienced by the components, and also by the rate of voltage rise.

In previous work [1], the authors used data on the impulse breakdown of a polymer-oil interface [2,3] to derive values of the mean statistical time $\overline{t_{s}}$ and the formative time $t_{f}$ for

Manuscript received on 8 September 2016, in final form 9 February 2017, accepted 20 February 2017. Corresponding author: M. J. Given. breakdown, assuming that the behavior of the system followed the von Laue distribution [4], as shown in Figure 1.

In the basic von Laue approach, it is assumed that: $\overline{t_{s}}$ is associated with the initiation of the discharge across the gap and has an exponential distribution, while $t_{f}$ describes the progress of the discharge across the gap and collapse of the gap voltage and is constant. For such behavior, a plot of minus the natural $\log$ of the survival function $S(t)$ vs. $t$ should produce a linear plot with gradient equal to the inverse of the value of $\bar{t}_{s}$, and which crosses the time axis at the value of $t_{f}$. Such plots are referred to as Laue plots or Lauegrams.

When experimental data is plotted on a Laue plot in practice, it is frequently observed to deviate from the expected linear behavior at short breakdown times. This has been ascribed to the 
influence of a distribution in the formative time which is assumed to be normal and independent of the distribution in the statistical time [5, 6]. When dealing with such plots, it is assumed that the values of $t_{f}$ and $\overline{t_{s}}$ derived from the linear sections of the graph are valid as long as the standard deviation of the formative time $\sigma_{f}$ is much smaller than the value of the statistical time $\overline{t_{s}}$.

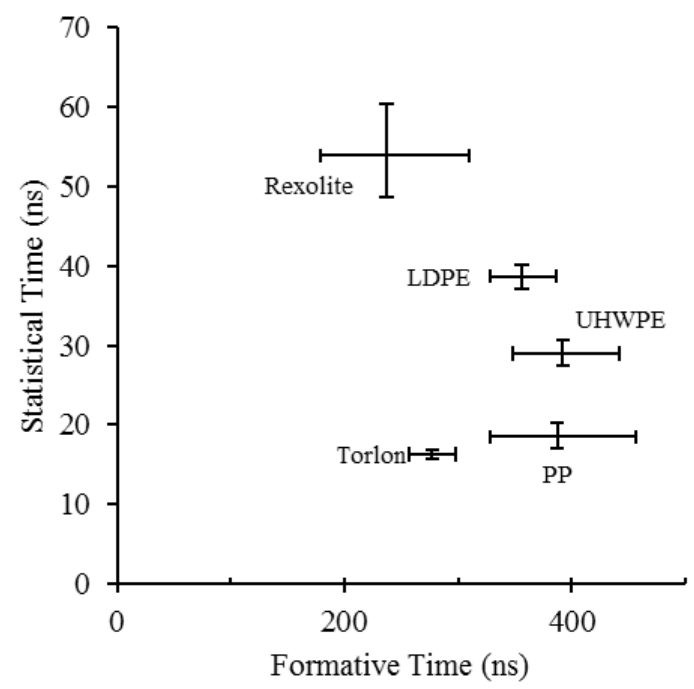

Figure 1. Plot of statistical and formative times derived from von Laue analysis for different polymers [1].

Although this approach was used by the authors [1] to analyze the data for breakdown along the interface between polymer and liquid insulation, upon reflection the authors consider that there are weaknesses in the approach: Firstly, although error bars are shown in Figure 1, these only reflect the uncertainty in the fit to the straight line section of the graph, and give no information in terms of the confidence in the fit; secondly, no value had been determined for $\sigma_{f}$, so that it was not possible to determine if the criterion that $\sigma_{f}<\bar{t}_{s}$, required to derive valid values of $t_{s}$ and $t_{f}$ had been met; finally, either certain points from the data sets had been excluded, or a limited number of points had been used to achieve straight line plots. The justifications for the decisions on which data points were included were reported in [1]. Therefore, this paper reports a more rigorous approach to fitting the data, assuming that the data is a result of an exponential distribution of the statistical time, as assumed by von Laue, coupled with a normally-distributed formative time.

\section{EXPERIMENTAL DATA USED FOR FITS}

The experimental data was obtained using a $1 / 6.5 \mu$ s waveshape, with a peak voltage of $-350 \mathrm{kV}$. The polymer samples were cylindrical with a height of $11.5 \mathrm{~mm}$ and a diameter of $25 \mathrm{~mm}$. Five different types of polymer were used as samples: Polypropylene; LDPE; UHMWPE; Rexolite; and Torlon. The samples were machined to the required shape and size as described in [2, 3] using a lathe. No additional polishing or surface roughening treatments were performed after machining. Before use the samples were cleaned and dried following the protocol described in [2]. For tests, samples were held between two stainless steel plane electrodes $(50 \mathrm{~mm}$ diameter $)$ and immersed in L10B mineral oil (EOS Ltd.) for testing at ambient pressure and temperature $\left(\sim 22^{\circ} \mathrm{C}\right)$. In order to reflect industrial conditions, the oil was used as received from the supplier, i.e. no degassing or de-humidifying was conducted. The high-voltage electrode included a collar to encourage breakdown across the surface of the polymer. The form of this electrode, a full description of the experimental system, the test protocol, and typical waveforms are reported in $[2,3]$.

The electrical properties of the materials used, based on [711] and the measured surface impulse breakdown fields from [2] are shown in Table 1. Note the bulk breakdown data is based on short time tests rather than on impulse tests.

Table 1. Electrical Properties of Polymer Materials Used

\begin{tabular}{c|c|c|c|c|c}
\hline \multicolumn{2}{c|}{} & \multicolumn{2}{c|}{ Resistivity } & Dielectric strength \\
\hline \hline Material & $\varepsilon_{\mathrm{r}}$ & $\begin{array}{c}\text { Surface } \\
(\Omega / \mathrm{sq})\end{array}$ & $\begin{array}{c}\text { Volume } \\
(\Omega \mathrm{cm})\end{array}$ & $\begin{array}{c}\text { Bulk } \\
(\mathrm{kV} / \mathrm{cm})\end{array}$ & $\begin{array}{c}\text { Surface } \\
(\mathrm{kV} / \mathrm{cm})\end{array}$ \\
\hline \hline PP & 2.25 & $10^{13}$ & $10^{16}-10^{18}$ & $200-260$ & $637 \pm 58$ \\
\hline LDPE & 2.2 & $10^{13}$ & $10^{15}-10^{18}$ & $180-270$ & $631 \pm 146$ \\
\hline UHMWPE & 3.0 & $>10^{13}$ & $>10^{14}$ & 350 & $483 \pm 64$ \\
\hline Rexolite & 2.5 & $>10^{14}$ & $>10^{16}$ & 200 & $503 \pm 70$ \\
\hline Torlon & 4.2 & $>10^{13}$ & $>10^{14}$ & 230 & $450 \pm 38$
\end{tabular}

\section{INFLUENCE OF A NORMALLY- DISTRIBUTED FORMATIVE TIME ON THE VON LAUE DISTRIBUTION}

To determine the influence of a normal distribution of the formative time on the values of $t_{f}$ and $\bar{t}_{s}$ derived from a Laue plot, simulations were run in the Maple 15 mathematics and modeling software produced by MapleSoft. This package includes the functionality to produce random samples of defined distributions. A 200-point sample of predicted time to breakdown initiation was formed using an exponential distribution governed by a value $t_{s m}$. A 200-point sample of predicted time between initiation and completion of breakdown was then formed using a normal distribution governed by a mean value of $t_{f m}$ and a standard deviation value $\sigma_{f m}$. The time values in these two samples were then added to produce a 200 point sample modelling a total time to breakdown. This sample was then sorted into ascending order and the corresponding values of:

$$
-\ln (S(t))=-\ln \left(\frac{N(t)}{N}\right)
$$


were calculated for each breakdown time in the sample. This process produced a data set equivalent to that which would be used to produce a Laue plot. To simulate finding the line of best fit using longer times to breakdown, where the influence of the distribution of formative times is assumed to be small, the equation of the straight line of best fit for the 20 pairs of values of $(t,-\ln (S(t))$ with the highest values of $t$ was then determined. The equation of this fit was then used to calculate values for the derived statistical time $\overline{t_{s d}}$ and the formative time $t_{f d}$ using the standard Laue plot approach.

This process was repeated 1000 times for any given set of values of the modeling parameters $t_{s m}, t_{f m}$ and $\sigma_{f m}$, resulting in 1000 pairs of values for $\overline{t_{s d}}$ and $t_{f d}$. Examining the distributions of values derived for $\overline{t_{s d}}$ and $t_{f d}$, it was found that they failed the Shapiro-Wilk normality test, therefore instead of describing the derived data using the mean and standard deviation of the distribution, the median 'value and the median average deviation (MAD) were calculated. By deriving the cumulative probability function $F_{T}(t)$ for the distribution, it was also possible to identify two additional parameters: $t_{16}$ and $t_{84}$, the values of $t$ where $F_{T}(t)$ was equal to 0.16 and 0.84 , respectively. For a normal distribution, the parameters $t_{16}$ and $t_{84}$ would approximate to the values one standard deviation below and above the mean value. The "jitter" in breakdown and switching operations is frequently defined by the standard deviation of breakdown times, assuming that the distribution is normal. For the non-normal distributions observed in these simulations, the parameters $t_{16}$ and $t_{84}$ would approximate to the values one standard deviation above and below the median to provide an equivalent representation of the "jitter" for the distributions.

Figures $2 \mathrm{a}$ and $2 \mathrm{~b}$ show the behavior of the derived statistical and formative times based on data generated for a statistical time $t_{s m}=50$ and formative time $t_{f m}=100$, as the standard deviation of the formative time $\sigma_{f m}$ was varied between 0 and 90 .

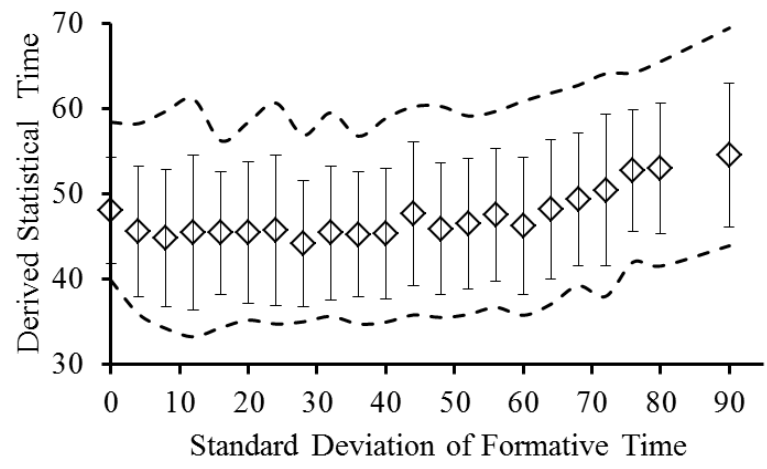

Figure 2a. Effect of standard deviation of formative time $\sigma_{f m}$ on derived values of statistical time $\overline{t_{s d}}$ for: $t_{s m}=50 ; t_{f m}=50$. Error bars MAD, dotted lines $t_{16}$ and $t_{84}$.

It can be seen in Figure 2a that there is little change in the behaviour of the derived statistical time $\overline{t_{s d}}$ as the standard deviation associated with the actual formative time increases, while $t_{s m} \geq \sigma_{f m}$. However, the median value for the distribution of derived values for the statistical time falls below the value of statistical time used to generate the data sets.

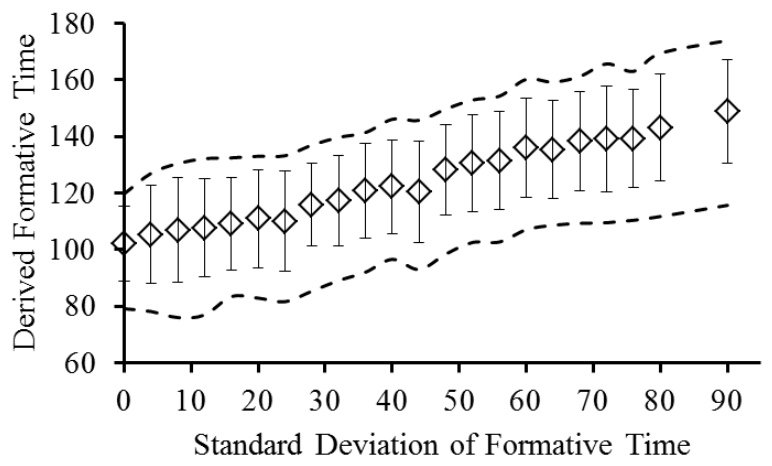

Figure 2b. Effect of standard deviation of formative time $\sigma_{f m}$ on derived values of formative time $t_{f d}$ for: $t_{s m}=50 ; t_{f m}=50$. Error bars MAD, dotted lines $t_{16}$ and $t_{84}$.

As the value of $\sigma_{f m}$ increases and exceeds $t_{s m}$, the derived values for $\overline{t_{s d}}$ start to show an upward trend. The derived values of the formative time $t_{f d}$ show a different behaviour where the median value increases steadily as the value of $\sigma_{f m}$ is increased, Figure 2b.

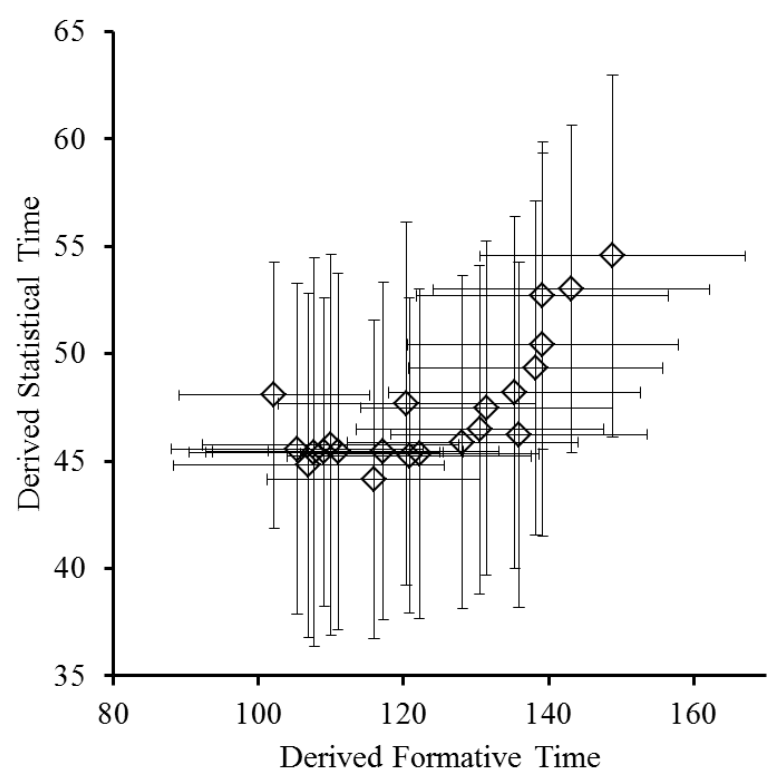

Figure 2c. Plot of derived values of statistical time $\overline{t_{s d}}$ and formative time $t_{f d}$ as standard deviation of formative time $\sigma_{f m}$ varied between 0 and 90 for: $t_{s m}=$ $50 ; t_{f m}=100$. Error bars MAD.

The effect of the change in the behaviour of the derived statistical time can be seen more clearly in Figure 2c. A similar behaviour has been observed for a large range of values of $t_{s m}$ 
and $t_{f m}$, with the derived statistical time remaining relatively constant, but below the true value, while $\sigma_{f m}$ is less than $t_{s m}$, while the derived formative time increases with $\sigma_{f m}$.

\section{FITTING TO EXPERIMENTAL DATA}

The appearance of the Laue plots of the experimental data reported in [1] and [2] indicated that the formative time was not constant but had a distribution of values, assumed to be normal. This, coupled with the results of the simulations reported in Section 3, indicated that the simple approach of using a linear fit would not result in an accurate estimation of the value of the formative time, and it was possible that errors would also occur in the derived value of the statistical time. To derive values for $\bar{t}_{s}$ and $t_{f}$ with a known degree of confidence, it was therefore decided to use the Kolmogorov-Smirnov (KS) statistical approach to fit the data to a von Laue distribution, modified with a normally-distributed formative time. This approach was selected as the KS approach permits tests on arbitrary distributions.

\subsection{FITTING APPROACH BASED ON KS STATISTICS}

Kolmogorov-Smirnov statistics allow the comparison of a data set with an arbitrary distribution function or a second data set. The approach compares the cumulative probability distribution functions (CDF) of the two data sets, and the KS statistic is the maximum difference between the values of the two cumulative distributions.

In the case of fitting a simple von Laue distribution where the $\mathrm{CDF}$ is defined at times $t \leq t_{f}$ by:

$$
F_{\mathrm{T}}(t)=\frac{N(t)}{N_{0}}=1-\exp \left(-\frac{t-t_{f}}{\overline{t_{s}}}\right)-1
$$

The effect of the formative time $t_{f}$ is to displace the CDF without changing its shape. Therefore, if both the CDF for the experimental data $F_{T E x}(t)$ and the CDF of the test function $F_{\text {TTest }}(t)$ are shifted in time so that:

$F_{\mathrm{TEx}}^{\prime}(0)=F_{\mathrm{T} T e s t}^{\prime}(0)=0.5$

where:

$$
F_{\mathrm{T}}^{\prime}(t)=F_{\mathrm{T}}(t+\tau)
$$

this allows the fit for the value of $\overline{t_{s}}$ to be performed independently of the value associated with $t_{f}$. Once the optimal value of $\overline{t_{s}}$ has been derived, the value of $t_{f}$ can then be obtained, by calculating the difference between $t_{50}$ for the experimental distribution $F_{T E x}(t)$ and a distribution derived from the optimal values of $\bar{t}_{s}, F_{\text {TFit }}(t)$ :
$F_{\text {TFit }}(t)=1-\exp \left(-\frac{t}{\overline{t_{s}}}\right)$

This approach has the advantage of reducing the search-space for the fitting parameters to a one-dimensional space in statistical time $\left(\overline{t_{s}}\right)$.

When dealing with a system where the formative time is not constant, but is normally distributed around a mean value $\overline{t_{f}}$ with a standard deviation $\sigma_{f}$, the distribution of formative times is the normal distribution with mean 0 and standard deviation $\sigma_{f}$, shifted in time by the mean value of the formative time $\overline{t_{f}}$. This allows the approach described above, where the KS tests are performed on time-shifted CDFs following equation $3 \mathrm{~b}$, to again be performed, reducing the search-space for the fitting process to two dimensions $\left(\bar{t}_{s}\right.$ and $\left.\sigma_{f}\right)$. Once the optimal values have been derived for these two parameters, the required value of $\overline{t_{f}}$ can then be calculated.

\subsection{DERIVING PARAMETER VALUES}

Software was developed in Maple 15 to allow the generation of test fit data based on either a simple exponential von Laue distribution, or on a distribution where the exponential von Laue distribution was combined with a normally-distributed formative time, following the procedure described in Section 3. The goodness-of-fit of this test data could then be assessed using the KS approach through Maple functions based on those available within the KSNStat package [12]. The code in this package was based on a Fortran GSMIRN algorithm [13]. For each set of experimental data, a search-space of possible values of $\overline{t_{s}}$ was explored for the simple von Laue fitting process and one of possible values of $\overline{t_{s}}$ and $\sigma_{f}$ for the combined exponential and normal fit.

Due to the relatively small number of samples in the experimental data sets (35), generally a cluster of data points in the search space would return the maximum value of the $\mathrm{KS} p$ parameter indicating closeness of match. Therefore, the median values and the median average deviation were calculated for this cluster.
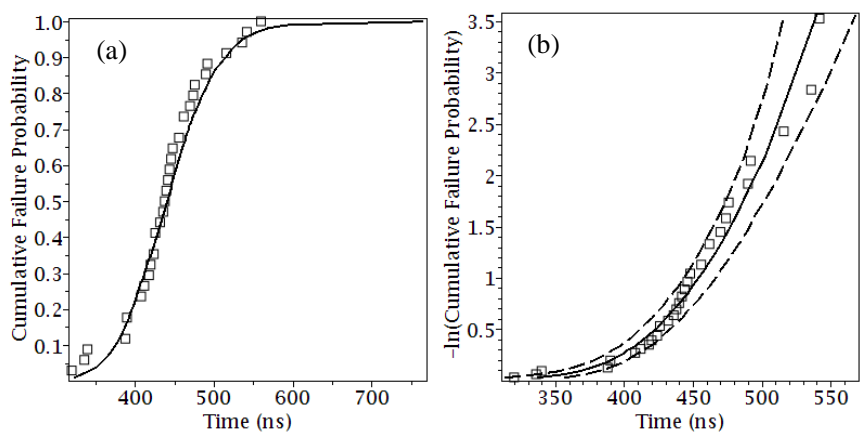

Figure 3a. $\mathrm{CDF}$ for optimal fit to polypropylene data using combined exponential and normal distributions: points experimental data; line optimal fit. b. Corresponding von Laue plot: points experimental data; solid lines median values; dotted lines $t_{16}$ and $t_{84}$. 
Figure $3 \mathrm{a}$ shows the CDF of the experimental data for polypropylene and that of the optimal fit based on median values of $\overline{t_{s}}$ and $\sigma_{f}$ from the fitting cluster and the subsequentlycalculated value of $\bar{t}_{f}$. Figure $3 \mathrm{~b}$ shows the von Laue plot for this data. To derive the von-Laue behavior based on the parameters derived from the fitting process, 1000 random samples, each containing 35 points, were generated in Maple using the median values of $\overline{t_{s}}, \overline{t_{f}}$ and $\sigma_{f}$ derived from the fitting process. For each value of $-\ln (S(t))$, the median value for the time to breakdown $t_{B d}$ (shown as the solid line in Figure $3 \mathrm{~b}$ ) and the values of $t_{16}$ and $t_{84}$ (shown as dotted lines in Figure $3 \mathrm{~b})$ were calculated. It was not possible to achieve acceptable fits to the experimental data using only an exponential distribution combined with a constant formative time. Therefore, in this paper, the results of fits to combined exponential and normal distributions are reported only.

\subsection{FITS OF EXPERIMENTAL DATA}

From the plots in Figure 3, it can be seen that a reasonable fit to the experimental data has been achieved for polypropylene. The maximum value of the KS $p$ parameter returned by the fitting process for polypropylene was 0.921 for parameter values in the range: $\overline{t_{s}}=34 \pm 3.0 n s, \overline{t_{f}}=408 \pm 3.8 \mathrm{~ns}$ and $\sigma_{f}=$ $44 \pm 1.4 \mathrm{~ns}$. Here the quoted value is the median and the range represents the MAD value calculated for the parameter from the cluster in the search space.

Figure 4 shows the corresponding plots for the breakdown data for LDPE. In this case, the KS $p$ parameter reached a maximum of 0.707 during fitting, and the optimal parameters of the fits were derived as: $\overline{t_{s}}=34 \pm 1.5 n s, \overline{t_{f}}=353 \pm 3.5 \mathrm{~ns}$ and $\sigma_{f}=26 \pm 3.1 \mathrm{~ns}$. However, from Figure $4 \mathrm{~b}$, it can be seen that while the data for times less than $400 \mathrm{~ns}$ fits the combination of exponential and normal distributions well, there is a significant deviation occurring with the results at longer times, with experimental data falling below the $t_{16}$ boundary derived for the fit parameters. In the previous analysis [1], it was noted that these points were associated with the initial measurements of breakdown behavior, and it was felt that some type of conditioning may have been occurring.
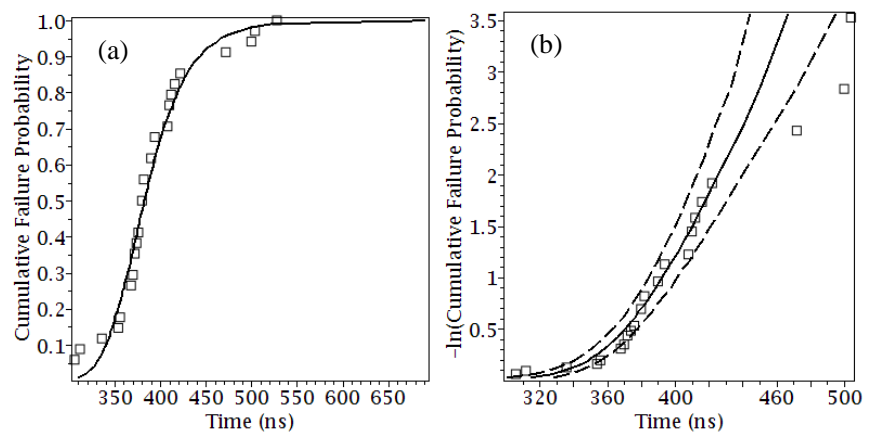

Figure 4a. CDF for optimal fit to LDPE data using combined exponential and normal distributions: points experimental data; line optimal fit. b. Corresponding von Laue plot: points experimental data; solid line median values; dotted lines $t_{16}$ and $t_{84}$.

Figure 5 shows the fitting results for UHMWPE, where the KS $p$ parameter for the optimal fit returned a value of 0.707 . The derived values for the optimal fit parameters were: $\overline{t_{s}}=34 \pm$ $1.4 n s, \overline{t_{f}}=374 \pm 3.4 n s$ and $\sigma_{f}=9.0 \pm 2.3 n s$. Although the value of the KS $p$ parameter is similar to that obtained for LDPE, the data points in general fall within or close to the $t_{16}$ and $t_{84}$ boundaries. However, the CDF plot suggests that the fit is poor for values at times greater than $400 \mathrm{~ns}$. On the von Laue plot in this range, all but one of the experimental points fall below the line derived for the median behavior of the fitted data. The combination of the value of $p$ and the appearance of the von Laue plot suggests that it is possible that more than one breakdown mechanism is occurring.

Figure 6 shows the fitting plots for the breakdown of Rexolite where the value of the KS $p$ parameter is again 0.707. The optimal fitting parameters for Rexolite are: $\bar{t}_{s}=108 \pm 6.8 \mathrm{~ns}$, $\overline{t_{f}}=100 \pm 6.9 \mathrm{~ns}$ and $\sigma_{f}=59 \pm 4.2 \mathrm{~ns}$. For this material, again the majority of the experimental data lies within or close to the $t_{16}$ and $t_{84}$ boundaries. However, the relatively low value of the parameter $p$ and the appearance of both the CDF and the von Laue plot at breakdown times greater than $250 \mathrm{~ns}$ suggest that again perhaps more than one breakdown mechanism may be occurring.
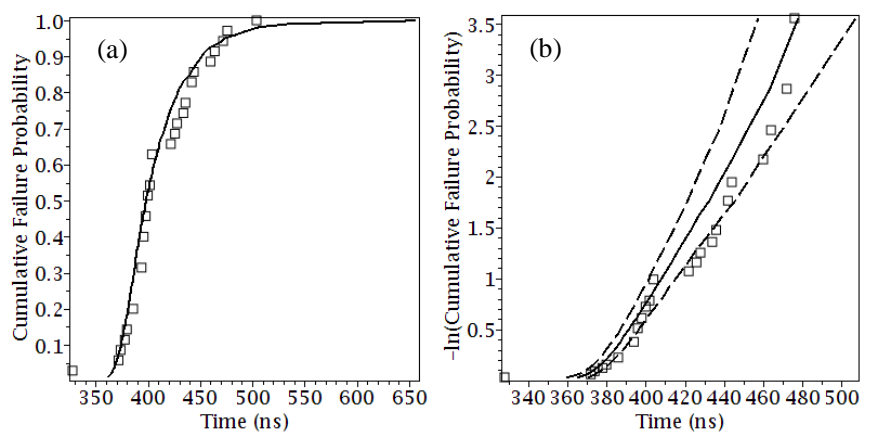

Figure 5a. CDF for optimal fit to UHMWPE data using combined exponential and normal distributions: points experimental data; line optimal fit. b. Corresponding von Laue plot: points experimental data; solid line median values; dotted lines $t_{16}$ and $t_{84}$. 

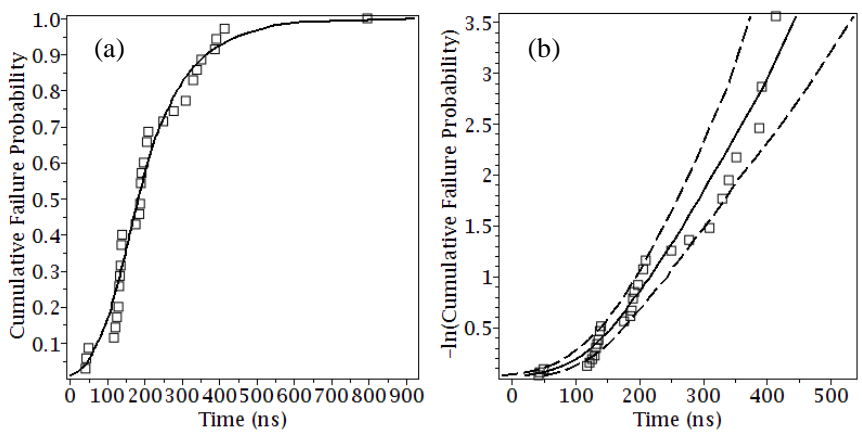

Figure 6a. CDF for optimal fit to Rexolite data using combined exponential and normal distributions: points experimental data; line optimal fit. b. Corresponding von Laue plot: points experimental data; solid line median values; dotted lines $t_{16}$ and $t_{84}$.

(a)

(b)

\begin{tabular}{c|c|c|c|c|c|c}
\hline \hline & \multicolumn{3}{|c|}{ Simple Laue fit to selected data [1] } & \multicolumn{3}{|c}{ Laue fits with standard deviation in formative time } \\
\hline \hline Material & $\bar{t}_{s}(n s)$ & $t_{f}(n s)$ & $\bar{t}_{s}(n s)$ & $\bar{t}_{f}(n s)$ & $\sigma_{f}(n s)$ & $p_{K S}$ \\
\hline \hline PP & $55.0+2.8 /-2.6$ & $343+39 /-35$ & $34 \pm 3.0$ & $408 \pm 3.8$ & $44 \pm 1.4$ & 0.921 \\
\hline LDPE & $38.7+1.5 /-1.4$ & $356+30 /-26$ & $34 \pm 1.5$ & $353 \pm 3.5$ & $26 \pm 3.1$ & 0.702 \\
\hline UHMWPE & $28.0+1.7 /-1.5$ & $392+49 /-45$ & $34 \pm 1.4$ & $374 \pm 3.4$ & $9 \pm 2.3$ & 0.702 \\
\hline Rexolite & $53.9+7.5 /-6.2$ & $237+72 /-57$ & $108 \pm 6.8$ & $100 \pm 6.9$ & $59 \pm 4.2$ & 0.702 \\
\hline Torlon & $16.3+0.6 /-0.5$ & $277+21 /-20$ & $71 \pm 4.8$ & $171 \pm 6.4$ & $55 \pm 4.5$ & 0.281 \\
\hline \hline
\end{tabular}

Figure 7 shows the fitting plots for the Torlon experimental data. The derived parameters were: $\overline{t_{s}}=71 \pm 4.8 \mathrm{~ns}, \overline{t_{f}}=$ $171 \pm 6.4 n s$ and $\sigma_{f}=55 \pm 4.5 n s$. The value of the KS $p$ parameter of 0.281 for this data indicates that the data cannot be fitted to a combination of exponential and normal distributions. This is supported by the fitting plots of the data.

The derived values of the parameters for this fitting process are shown in Table 2, which also summarizes the values derived using simple Laue plots, reported in [1]
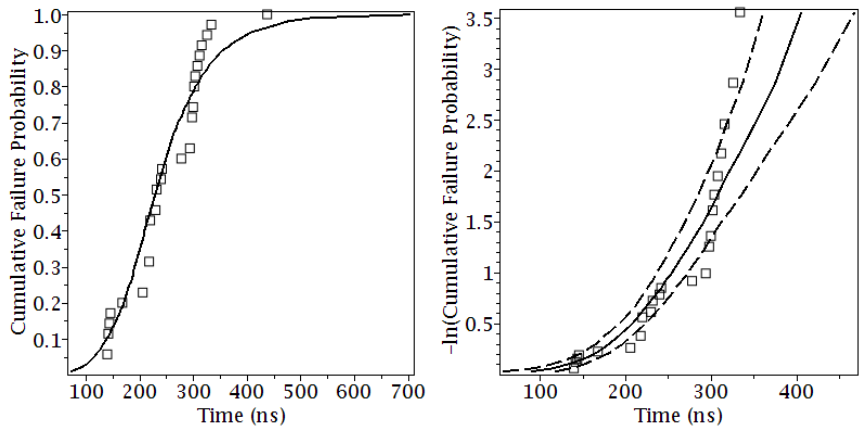

Figure 7a. CDF for optimal fit to Torlon data using combined exponential and normal distributions: points experimental data; line optimal fit. b. Corresponding von Laue plot: points experimental data; solid line median values; dotted lines $t_{16}$ and $t_{84}$.

\section{DISCUSSION}

It can be seen that, for LDPE and UHMWPE, the values of $\overline{t_{s}}$ derived using the combined exponential and normal distributions are similar to the values reported in the earlier analysis [1]. This is consistent with the analysis of the influence of a normal distribution of the formative time on the derived values of statistical time in Section 3 of this paper, as the derived values of standard deviation in the formative time $\sigma_{f}$ for these polymers is less than the statistical time $\overline{t_{s}}$. For polypropylene, the value of $\overline{t_{s}}$ derived in [1], ignoring the effect of the normal distribution in the formative time, is considerably larger than that derived taking into account the normal distribution; this is, again, consistent with the analysis in Section 3, as the value of the standard deviation of the formative time $\sigma_{f}$ is larger than the value of the statistical time $\overline{t_{s}}$.

The values for the statistical times derived taking into account a normal distribution of the formative time are very similar in value for polypropylene, LDPE and UHMWPE. Compared to the results reported in [1], there is also a clear separation in the behavior of the statistical time for polypropylene, LDPE and UHMWPE and that for the remaining two polymers: Rexolite and Torlon. A clear separation in the behavior of the formative time is also clearly observable between these two groups of polymers with longer formative times associated with polypropylene, LDPE and UHMWPE. There is also a suggestion that the standard deviation of the formative time is larger for Rexolite and Torlon as compared with the other polymers.

The fits of the experimental data to an exponential distribution of statistical times combined with a normal distribution of formative times may not be adequate for the majority of the polymers considered. This supports the suggestion made in [1] that more than one breakdown mechanism each associated with a formative and statistical time may have occurred during the experimental tests. In preliminary simulations of the expected behavior of time to breakdown in a system where two different breakdown mechanisms occurring, 
each with a separate statistical and formative time, von Laue plots with a similar form to those shown for UHMWPE in Figure $5 \mathrm{~b}$ and Rexolite in Figure $6 \mathrm{~b}$ have been observed.

The authors have considered fitting their experimental data to simulated data based on two combinations of an exponentiallydistributed statistical time and a normally-distributed formative time. The difficulties with this approach would be determining the relative frequency of occurrence of the two breakdown mechanisms, coupled with an expansion of the search-space from three dimensions $\left(\overline{t_{s}}, \overline{t_{f}}, \sigma_{f}\right)$ reduced to two dimensions by offsetting the CDF to eliminate $\bar{t}_{f}$, to a five-dimensional searchspace $\left(\overline{t_{s 1}}, \overline{t_{f 1}}, \overline{t_{s 2}}, \overline{t_{f 2}}, \sigma_{f}\right)$, where: $\overline{t_{s 1}}$ and $\overline{t_{f 1}}$ are the mean statistical and formative times for the first breakdown mechanism; $\overline{t_{s 2}}$ and $\overline{t_{f 2}}$ are the mean statistical and formative times for the second breakdown mechanism; and $\sigma_{f}$ is the standard deviation in the formative time distributions, assumed to be the same for each distribution. This expansion in the dimension of the search-space will lead to a significant increase in the computational time required to optimize the fitting parameters. As there will be two parameters associated with formative times, the offsetting of the CDF used to reduce the dimension of the search-space in this paper will not be possible. This problem may be addressed by defining the formative time for the second breakdown mechanism $\overline{t_{f 2}}$ in terms of:

$\overline{t_{f 2}}=\overline{t_{f 1}}+\Delta t_{f}$

This approach would allow the offsetting of the CDF approach to again be used, eliminating $t_{f l}$ and allowing the use of a fourdimensional search space $\left(\overline{t_{s 1}}, \overline{t_{s 2}}, \Delta t_{f}, \sigma_{f}\right)$, with the values of $t_{f 1}$ and $t_{f 2}$ being calculated after the fit has been optimized.

However, fitting this double distribution based on only 35 data points will lead to a very large number of combinations of possible values of the five parameters considered, producing fits with equivalent confidence levels. This would result in very large clusters of equivalent optimal fits in the five-dimensional search space. In addition, the value for the relative frequencies of occurrence of the two breakdown mechanisms is effectively another parameter for the fit, and is likely to increase the size of the cluster in the search-space. It was, therefore, decided that there would be no benefit in attempting the double distribution fits with the data available.

\section{CONCLUSIONS}

A method for determining the values for the statistical and formative times for breakdown across the interface between polymer and oil insulators from experimental data has been implemented. In this method, it was assumed that the statistical time was exponentially distributed and the formative time was normally distributed.
It was shown that the presence of a normal distribution in the formative time should not significantly affect values of the statistical time derived from the straight-line section of a Laue plot, as long as the statistical time was larger than the standard deviation in the formative time. However, a reduction in the median value derived for the statistical time from fits to randomly generated samples was observed when compared to the underlying value used to generate the random distributions. Values for the formative time derived from the straight-line section of the Laue plots based on randomly generated samples were affected by the presence of any normal distribution in the formative time.

It was seen that fits to experimental data could be made in terms of a single breakdown mechanism, described by an exponentially-distributed statistical time and a normallydistributed formative time, for the majority of the materials examined. However, the values for the confidence of the fit and the appearance of the experimental and fitted Laue plots does not eliminate the possibility that more than one breakdown mechanism is

occurring.

\section{ACKNOWLEDGMENT}

Dr. Given would like to thank Dr. Robert Atkinson, a colleague in the Department of Electronic and Electrical Engineering at the University of Strathclyde, for suggesting the use of Kolmogorov-Smirnov statistics.

\section{REFERENCES}

[1] M. P. Wilson, M. J. Given, Igor V. Timoshkin, S. J. MacGregor, T. Wang, M.A. Sinclair, K. J. Thomas and J. M. Lehr; "The statistical and formative times for breakdown at a polymer-oil interface" IEEE Int'l. Power Modulator and High Voltage Conf.., pp. 43-46, 2012

[2] M. P. Wilson, S. J. MacGregor, M. J. Given, I. V. Timoshkin, M. A. Sinclair, K. J. Thomas, and J. M. Lehr, "Surface flashover of oil-immersed dielectric materials in uniform and non-uniform fields," IEEE Trans. Dielectr. Electr. Insul., Vol. 16, pp. 1028-1036, 2009.

[3] M. P. Wilson, M. J. Given, I. V. Timoshkin, S. J. MacGregor, M. A. Sinclair, K.J. Thomas and J. M. Lehr, "Impulse-Breakdown Characteristics of Polymers Immersed in Insulating Oil"; IEEE Trans. Plasma Sci., Vol. 38, No. 10, pp 2611-2619, 2010

[4] M. von Laue, "Bemerkung zu K. Zubers messung der verzögerungszeiten bei der funkenentladung", Ann. der Physik, Vol. 381, pp. 261-265, 1925.

[5] C. A. Maluckov, J. P. Karamarkovic, and M. K. Radovic, "Statistical analysis of electrical breakdown time delay distributions in neon tube at 13.3 mbar," IEEE Trans. Plasma Sci., Vol. 31, no. 6, pp. 1344-1348,Dec. 2003.

[6] C. A. Maluckov, J. P. Karamarković, M. K. Radović, and M. M. Pejović, "The application of convolution-based statistical model on the electrical breakdown time delay distributions in neon under $\gamma$ and UV radiation," IEEE Trans. Plasma Sci., Vol. 34, No. 1, pp. 2-6, 2006.

[7] The Plastic Shop Technical Data Sheet, PP, http://www.theplasticshop.co.uk/plastic_technical_data_sheets/fluted_poly propylene_technical_data_sheet.pdf, accessed $9^{\text {th }}$ August 2010.

[8] Goodfellow Data Sheet, Polymer-Electrical Properties, https://www.goodfellow.com/PDF/TAB301E.pdf, accessed $9^{\text {th }}$ August 2010. 
[9] Quadrant Engineering Plastic Products - Product Data Sheet, Ultra-High Molecular Weight Polyethylene, http://www.alperton.com/alperton/files/PDS_CESTILINE_HD1000_EN.p df, accessed $9^{\text {th }}$ August 2010.

[10] Ensinger Ltd. -Product Data Sheet Rexolite® 1422, http://www.ensingner.Itd.uk/1809.file.dld, accessed 9 ${ }^{\text {th }}$ August 2010.

[11] Quadrant Engineering Plastic Products - Product Data Sheet, Torlon 4203 PAI,http://www.theplasticshop.co.uk/plastic_technical_data_sheets/torlon_ 4203_technical_data_sheet.pdf, accessed $9^{\text {th }}$ August 2010.

[12] M.J Brown, "Generalised Smirnov two-sample homogeneity test"; http://www.maplesoft.com/applications/view.aspx?SID=121124. Accessed $17 / 01 / 2013$

[13] A. Nikiforov, "Exact Smirnov two-sample tests for arbitrary distributions", J. Appl. Stat., vol.43, No. 1. pp. 265-270, 1994

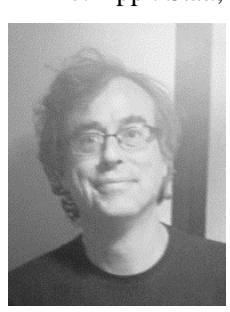

Martin J. Given (M'99-SM'11) is currently a Senior Lecturer in the Department of Electronic and Electrical Engineering at the University of Strathclyde. He received a degree in physics from the University of Sussex in 1981 and a Ph.D. in electronic and electrical engineering from the University of Strathclyde in 1996. He has a broad range of research interests. At present his interests include: ageing processes and condition monitoring in solid and liquid insulation, breakdown and conduction in liquids, solids and gasses; nano-composite materials; partial discharge behaviour at low pressures; bio-decontamination and long arcs and lightning attachment

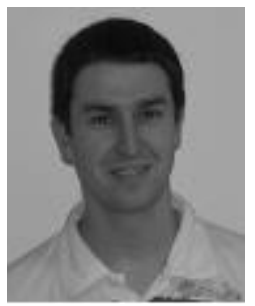

Mark P. Wilson (M'10) was born in Stranraer, Scotland, in 1982. He received the B.Eng. (with honours), M.Phil., and Ph.D. degrees in electronic and electrical engineering from the University of Strathclyde, Glasgow, U.K., in 2004, 2007, and 2011, respectively. He is presently based in the High Voltage Technologies research group at the University of Strathclyde, where his research interests include interfacial surface flashover, nanodielectrics, and the practical applications of high power ultrasound, corona discharges, and pulsed electric fields. Mark is a member of the IEEE Nuclear and Plasma Sciences Society, from whom he received a Graduate Scholarship Award in 2011, the IEEE Dielectrics and Electrical Insulation Society, and the IET.

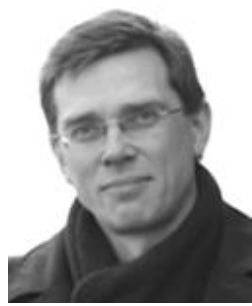

Igor V. Timoshkin (M'07-SM'14) received a degree in physics from the Moscow State University (Russia) in 1992, and the Diploma and the Ph.D. degree from the Imperial College of Science, Technology and Medicine, (London, UK) in 2001. After graduation from MSU he worked as a Researcher at Moscow State Agro-Engineering University, and then at the Institute for High Temperatures of Russian Academy of Sciences before moving to ICSTM in 1997. He joined the Department of Electronic and Electrical Engineering of the University of Strathclyde (Glasgow, UK) in 2001 where he became a Reader in 2016. His research interests include transient spark discharges in gases and liquids, environmental applications of non-thermal plasma discharges, plasma aircleaning and bio-decontamination technologies.

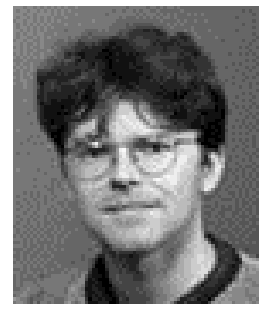

Mark Sinclair (M'03) received the B.Sc. degree from the Victoria University of Manchester, UK, in 1990 and then went to St. Andrews and Strathclyde Universities in Scotland to receive an M.Sc. in Laser Engineering and Pulsed Power Technology. In 1992 he joined the Pulsed Power Group at AWE. Initially he worked on the E Minor machine moving on to Mogul E which was optimized for radiography of dense objects. Currently he is the technical lead and Team Leader for Pulsed Power at AWE running a number of programs to develop fundamental knowledge and skills, to develop new x-ray sources and to use Pulsed Power for Hydrodynamic simulation.

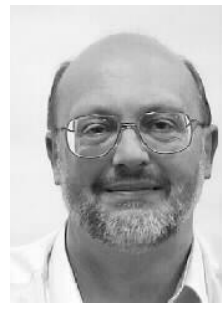

Kenneth J. Thomas (M'05-SM'10) was born in Carmarthen, South Wales in 1958. He received the B.Sc. degree in physics from Imperial College, London in 1979. He completed the Ph.D. degree in laser physics, also at Imperial College, in 1983 and joined the Pulsed Power Group at AWE. Since then he has been involved in the design and construction of multiMegaVolt pulsed power machines for flash radiography and supporting research, particularly the application of Induction Voltage Adder technology to the next generation of accelerators. He is a C. Phys. and a member of the IET and IoP

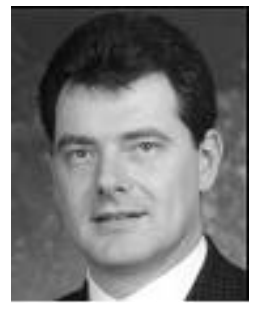

Scott J. MacGregor (M'95-SM'14) received the B.Sc. and Ph.D. degrees from the University of Strathclyde, Glasgow, U.K., in 1982 and 1986, respectively. He became a Pulsed Power Research Fellow in 1986 and a Lecturer in pulsed-power technology in 1989. In 1994, he became a Senior Lecturer, with a promotion to Reader and Professor of High Voltage Engineering, in 1999 and 2001, respectively. In 2006 and 2010 he became Head of the Department of Electronic and Electrical Engineering and Executive Dean of the Faculty of Engineering, and has been the Vice-Principal of the University of Strathclyde since 2014. Professor MacGregor was the recipient of the 2013 IEEE Peter Haas Award, and he was appointed as an Associate Editor of the IEEE Transactions on Dielectrics and Electrical Insulation in 2015. His research interests include high-voltage pulse generation, high-frequency diagnostics, high-power repetitive switching, high-speed switching, electronic methods for food pasteurization and sterilization, generation of high-power ultrasound (HPU), plasma channel drilling, pulsed-plasma cleaning of pipes, and stimulation of oil wells with HPU 


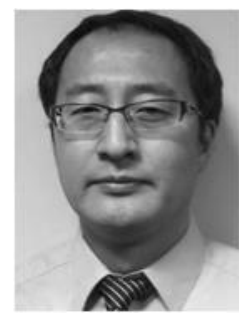

. Tao Wang received the B.Eng and M.Sc degrees from Northeast China Dianli University (China) in 1993 and 1996 respectively, and the Ph.D. degree from the University of Strathclyde (Glasgow, UK) in 2005. He then joined the Newland Entech as a research fellow developing high efficiency industrial ozone generator. He joined the department of Electronic and Electrical Engineering of University of Strathclyde as a lecturer in 2010. His research interests include non-thermal gas discharges and their applications in gas synthesis, water disinfection and advanced oxidation process in water

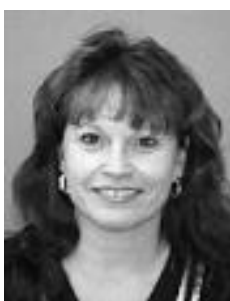

Jane Lehr (S'91-M'96-SM'02-F'08) received the B.Eng. degree from the Stevens Institute of Technology, Hoboken, NJ, and the Ph.D. degree in electro physics from Polytechnic University, New York, NY. She is currently a Professor in the Department of Electrical and Computer Engineering at the University of New Mexico Albequerque. Prior to this she has been a Member of the Technical Staff with the Exploratory Pulsed Power Technologies Branch, Sandia National Laboratories, Albuquerque, NM. andwas with the Directed Energy Directorate, Air Force Research Laboratory. Dr. Lehr is the Past President of the IEEE Nuclear and Plasma Sciences Society. 